J. Gynäkol. Endokrinol. AT 2019 · 29:28-32 https://doi.org/10.1007/s41974-019-0088-6

@ Der/die Autor(en) 2019

\section{Christoph Brezinka}

Universitätsklinik für gynäkologische Endokrinologie und Reproduktionsmedizin, Medizinische Universität Innsbruck, Innsbruck, Österreich

\title{
Der Dottersack - ein wichtiger Marker beim Ultraschall in der Frühschwangerschaft
}

Der Dottersack ist ein wenig beachteter Nebenbefund beim Ultraschall in der Frühschwangerschaft. Dieser wichtige Begleiter des winzigen Kindes übernimmt eine Zeitlang seine Blutproduktion, bevor er dann ab der 10. SSW langsam obliteriert. Im Ultraschall ist der Dottersack ein wichtiges Orientierungsmerkmal und eine diagnostische Hilfe.

Das allererste Zeichen einer intrauterinen Schwangerschaft, das wir im Ultraschall erkennen, ist die meist kreisrunde, echoarme Struktur, die im Ultraschalljargon „Fruchtsack“ genannt wird. Der Fruchtsack entsteht zwar durch den Embryo, er ist genau genommen ein vom mütterlichen Endometrium gebildetes „Drumherum“, in dem sich die Schwan- gerschaft entwickeln kann. Diagnostisch wichtig dabei ist der echodense Ring, die sogenannte deziduale Reaktion, die ein sehr verlässliches Zeichen dafür ist, dass es sich um eine topische Schwangerschaft handelt. Wirklich embryonalen Ursprungs ist die nächste Struktur, die wir einige Tage später erkennen können: der Dottersack („yolk sac“). Der im Ultraschall gerade erkennbare (sekundäre) Dottersack besteht aus den 3 Schichten Ektoderm, Mesoderm, Endoderm, wobei in der mesodermalen Schicht wichtige Vorgänge der Erythropoese stattfinden [4]. Der Prozess der Regression des Dottersacks beginnt ab der 10. SSW, nach der 12. SSW ist der Dottersack nur noch selten erkennbar [3]. Vereinzelt kann er auch bis ins zweite Trimenon bestehen und im Ultraschall erkennbar bleiben (vorausgesetzt, man untersucht sehr genau), dies hat aber keine ungünstigen Auswirkungen auf die Schwangerschaft [9]. In einem $8 \mathrm{~mm}$ großen Fruchtsack sollten wir mit einem guten Ultraschallgerät im Vaginalschall die Konturen eines Dottersacks erkennen können (• Abb. 1).

Wenige Tage später kann man den zunächst noch deutlich kleineren Embryo erkennen (-Abb. 2). Der Dottersack ist mit dem Embryo durch den Ductus omphaloentericus (Dottergang, engl. „,vitelline duct") verbunden (• Abb. 3). Dieser lässt sich gerade mit dem 3-D-Schall sehr schön darstellen (• Abb. 4).

Die genaue Messung des Dottersacks (Außenrand zu Außenrand) sollte Teil einer jeden Ultraschalluntersuchung in der Frühschwangerschaft sein. Es ist be-

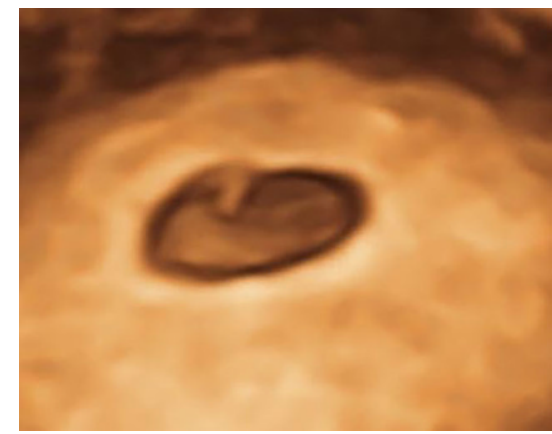

Abb. 1 In diesem $6 \mathrm{~mm}$ großen Fruchtsack lässt sich noch kein Embryo, aber eine Seite des Dottersacks im 3-D-Schall erkennen

Aktualisierung von: Brezinka C (2016) Der Dottersack in der Frühschwangerschaft. J Gynäkol Endokrinol 26(2):10-14

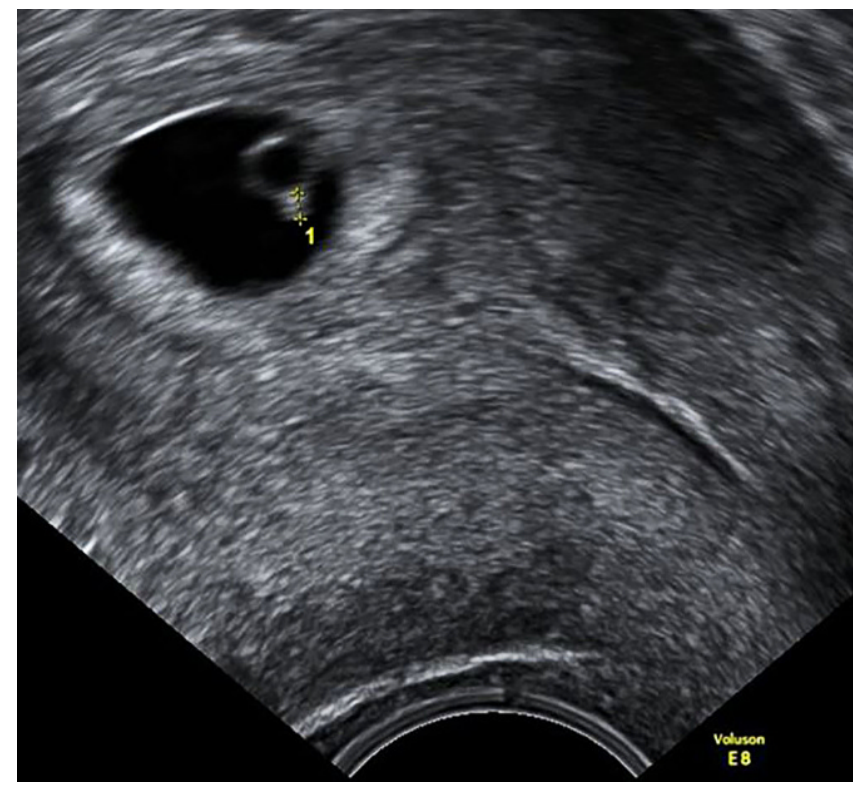

Abb. $2 \triangleleft$ Ein

$1,5 \mathrm{~mm}$ langer $\mathrm{Em}$ bryo neben einem $4 \mathrm{~mm}$ großen Dottersack. Herzaktionen sind bei diesem Embryo noch nicht zu erkennen. Die deziduale Reaktion um den Fruchtsack ist als markantes echodenses Band gut vom restlichen Endometrium zu unterscheiden 

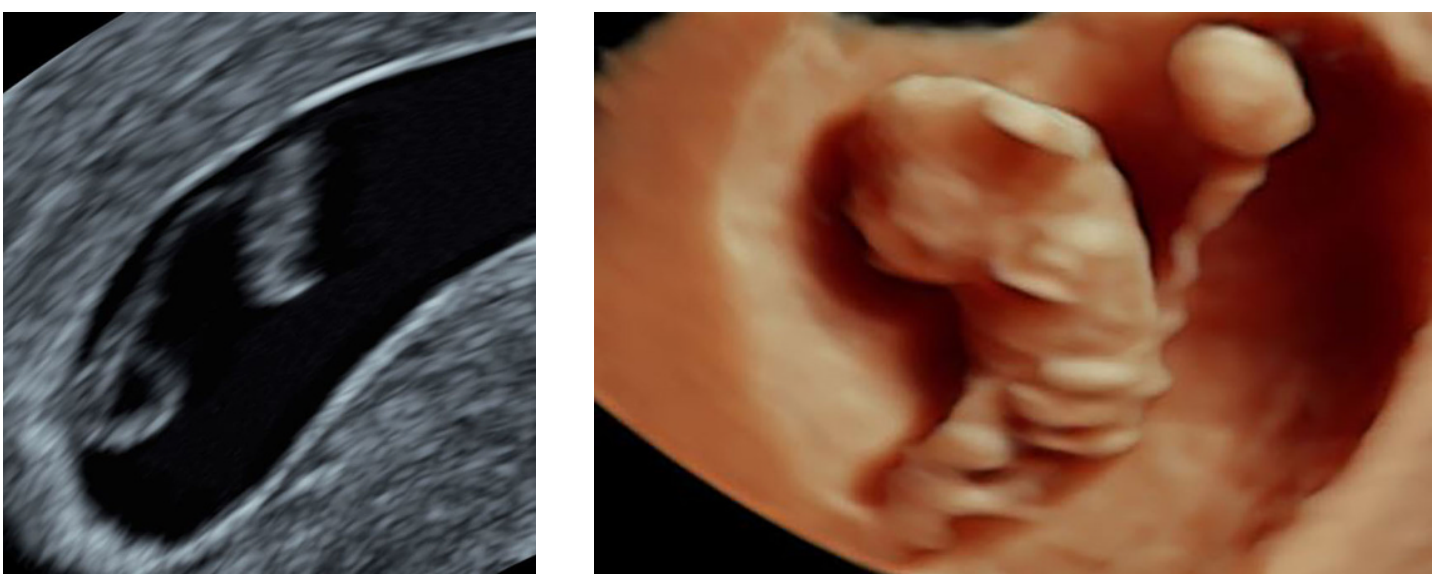

Abb. $4<$ Ein Embryo mit $17 \mathrm{~mm}$ Scheitel-Steiß-Länge und dem gut sichtbaren Ductus omphaloentericus im 3-D-Schall

Abb. $3 \Delta$ In einer Aufnahme aus der $7+0$. SSW zeigt sich, wie ein $4 \mathrm{~mm}$ großer Dottersack über den Ductus omphaloentericus mit einem vitalen $9 \mathrm{~mm}$ großen Embryo verbunden ist
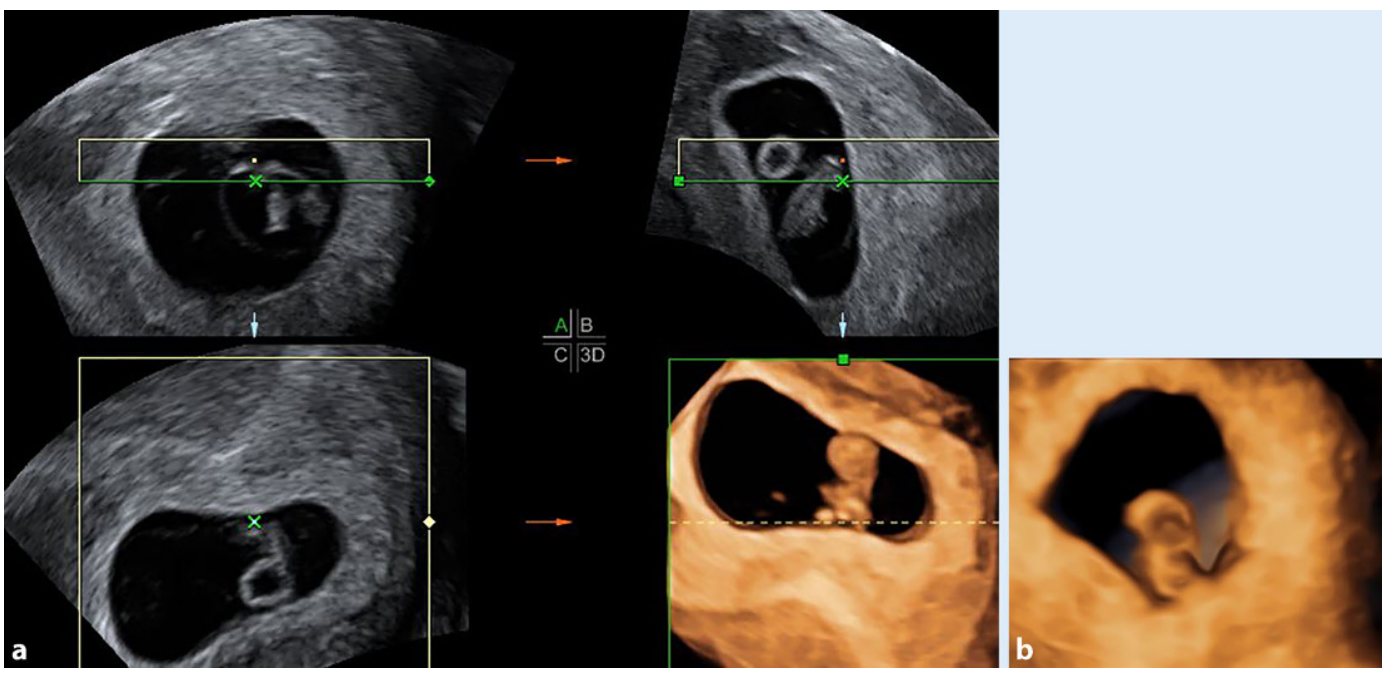

Abb. $5<$ a Vorsicht Messfehlergefahr! Liegen Embryo und Dottersack nahe beisammen, besteht stets die Gefahr, dass der Durchmesser des Dottersacks mit der Scheitel-Steiß-Länge des Embryos addiert wird (Bild unten rechts). $\mathbf{b}$ Vorsicht - Messfehlergefahr! 6+1.SSW: Der Dottersack liegt dem Embryo direkt an, die Versuchung ist groß, eine Messstrecke durch beide zu legen und damit einen großen Fehler zu produzieren

sonders darauf zu achten, dass der Embryo getrennt vom Dottersack vermessen wird. Meist liegt der Dottersack weit entfernt vom Embryo, dann ist dies kein Problem, es kann sich aber ergeben, dass der Dottersack direkt dem Embryonalpol anliegt und mit dem Kopf des Embryos verwechselt und daher mit ihm zusammen gemessen wird (• Abb. 5). Dies kann zu einer Fehlbestimmung des Gestationsalters um mehr als eine Woche führen und sollte unbedingt vermieden werden. Besonders anfällig ist hierfür die ansonsten so hilfreiche 3-D-Bildgebung.

Der Dottersack liegt immer außerhalb der Amnionhöhle (• Abb.6) Es dauert bis zur 14. SSW, manchmal bis zur 17. SSW, bis die Amnionhöhle so weit gewachsen ist, dass sie den gesamten intrakavitären
Raum ausfüllt. Bis dann sollte der Dottersack resorbiert sein.

Eine Rolle spielen die Dottersäcke bei der frühen Mehrlingsdiagnostik (-Abb. 7): Sieht man zwei Embryonen, aber nur eine Amnionhöhle und nur einen Dottersack, so handelt es sich wahrscheinlich um eine monochorialmonoamniale Schwangerschaft. Es gibt aber immer wieder Berichte, dass die weit verbreitete Faustregel „so viele Dottersäcke - so viele Embryonen" nicht immer zutrifft [2]. In der Praxis bedeutet dies, dass bei Mehrlingen der oder die beobachteten Dottersäcke in der Frühschwangerschaft stets schriftlich und mit Bildern dokumentiert werden sollten.

\section{Dottersack als Prognosefaktor}

Das völlige Fehlen eines Dottersacks in einer ansonsten intakt erscheinenden frühen Einlingsschwangerschaft ist ein prognostisch ungünstiges Zeichen. Sieht man innerhalb weniger Tage zwar einen Dottersack, aber keinen Embryo, so sollte die Schwangerschaft keinesfalls zu früh aufgegeben werden - ein Dottersack erhöht die Wahrscheinlichkeit, dass die Schwangerschaft mit Verzögerung doch noch als vital erkannt wird [5]

Seit den 1990er-Jahren gilt ein auffällig großer Dottersack (mehr als $6 \mathrm{~mm}$ Durchmesser) als ungünstiges prognostisches Zeichen für den weiteren Verlauf der Schwangerschaft [10]. Es gibt aber zahlreiche Berichte von normalen Schwangerschaftsverläufen und gesun- 


\section{Tipps \& Tricks im Gyn-Ultraschall}

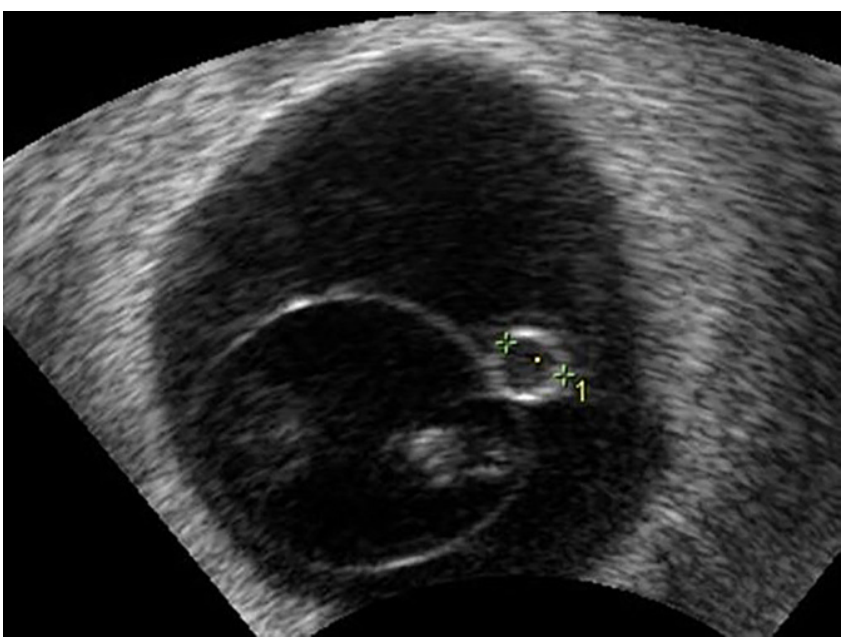

Abb. 6 A Embryo in der $6+5$. SSW innerhalb der Amnionhöhle, der Dottersack (5 mm im Querdurchmesser) ist immer außerhalb

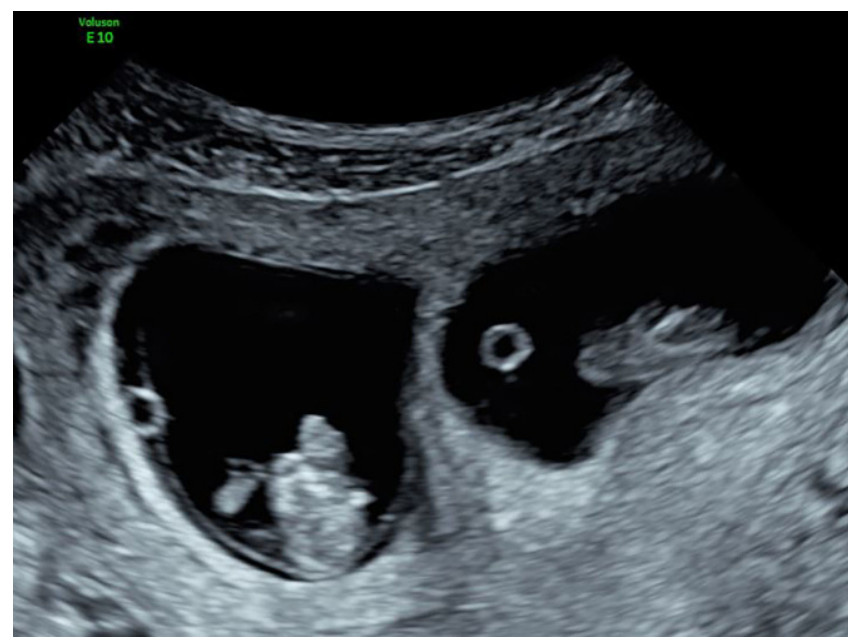

Abb. 7 ॥ 10 + 1. SSW - vitale Gemini im Abdominalschall. Es gelingt praktisch nie, beide Dottersäcke und beide Embryos im Längsschnitt in einer Ebene darzustellen. Markant ist in diesem Fall auch das "twin peaks sign“ („lambda sign“), das die Dichorionizität dieser Schwangerschaft zeigt
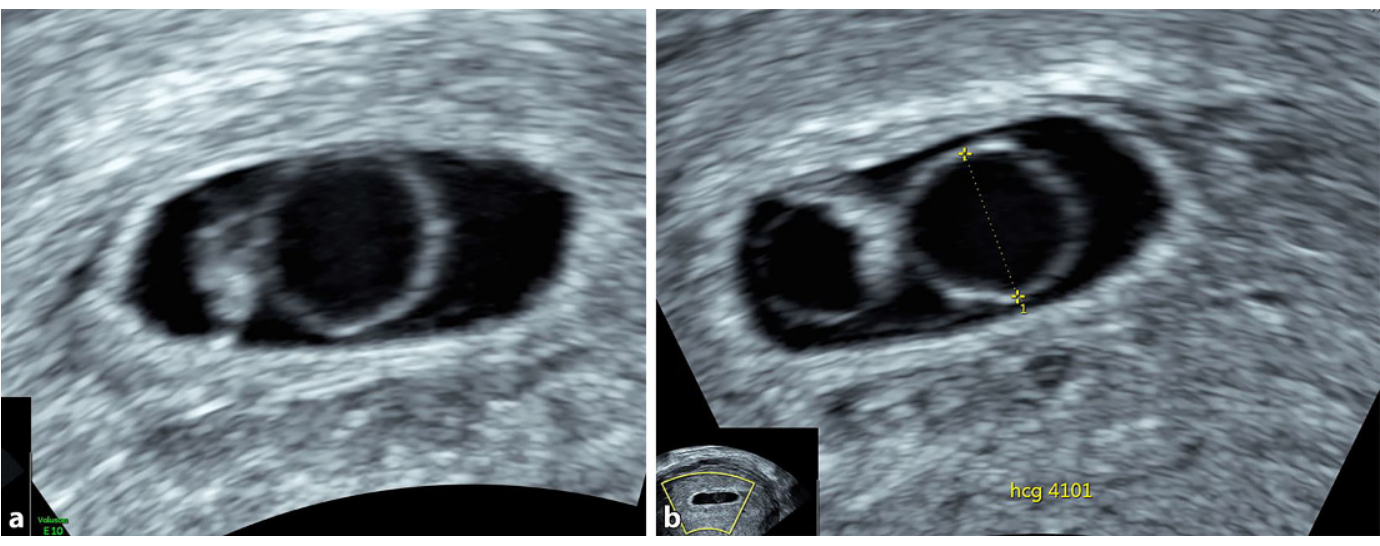

Abb. $8<$ a $7+4.5 S W$ vitaler Embryo mit 6,5 $\mathrm{mm}$ $\mathrm{CRL}$, auf $7 \mathrm{~mm}$ vergrößerter Dottersack, auffallend kleiner Fruchtsack. b Selber Fall, 5 Tage später: Der Embryo weist keine Herzaktion mehr auf, man sieht ihn links in der Amnionhöhle am Rand liegen, der Dottersack mit $8 \mathrm{~mm}$ wesentlich größer als die Amnionhöhle, der Fruchtsack insgesamt klein

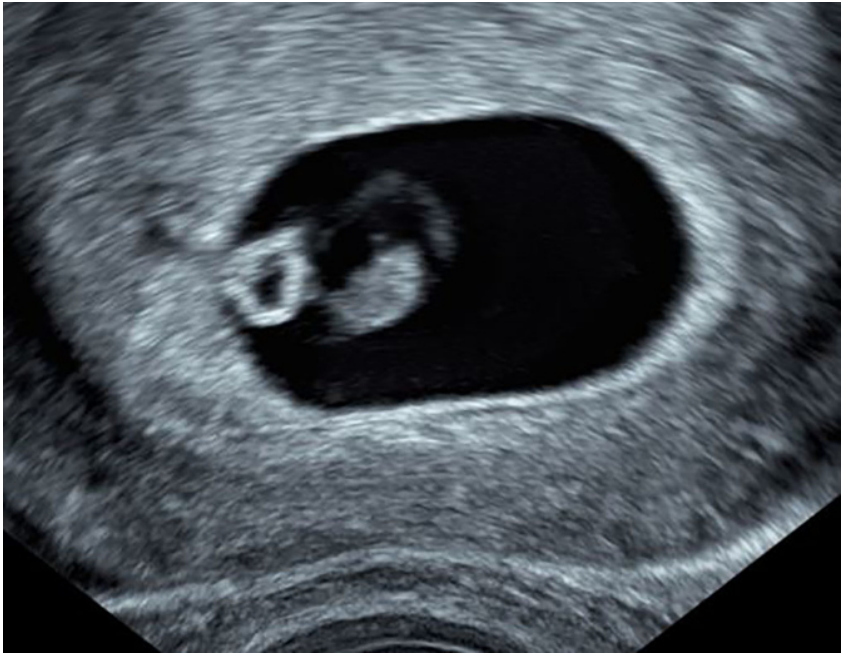

Abb. $9 \Delta$ In der 7 + 5. SSW zeigte sich ein etwas echodenser, dickwandiger und auch nicht ganz runder Dottersack. Die Schwangerschaft verlief weiter unauffällig

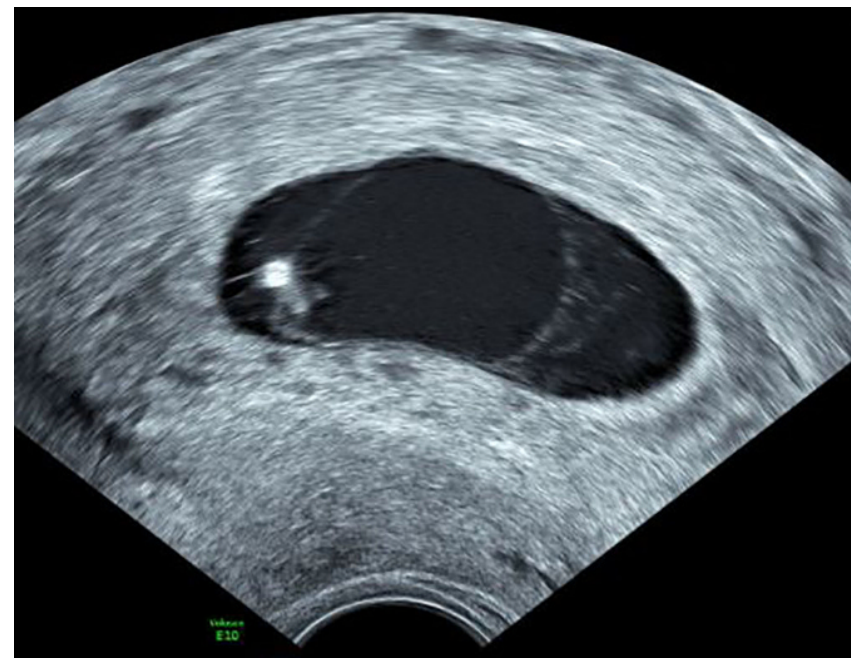

Abb. $10 \Delta$ Eine Schwangerschaft mit avitalem Embryo in der 8+3.SSW und einem auffallend echodensen Dottersack 


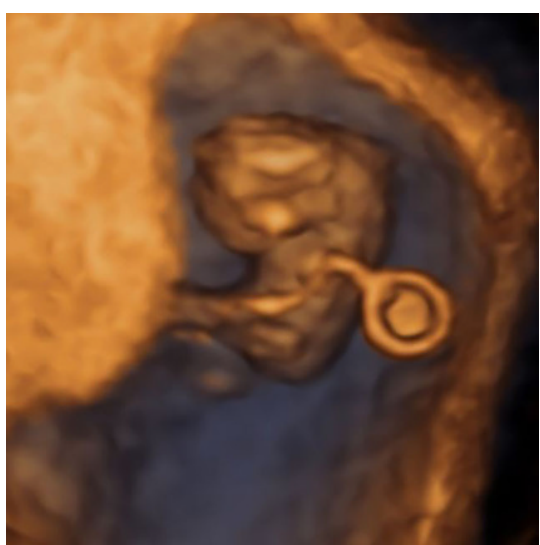

Abb. $11 \Delta 8+1$. SSW-im 3-D-Ultraschall wirkt der Dottersack wie eine Frisbeescheibe, die über den Ductus omphaloentericus mit dem Embryo verbunden ist

den Neugeborenen, bei denen in der Frühschwangerschaft ein auffällig großer Dottersack beobachtet wurde. In manchen Studien war es nicht der auffällige Durchmesser des Dottersacks, sondern sein Aussehen, das sich als prognostisch für ein Abortgeschehen erwies [7]. Aus diesem Grund sollten auch bei großem Dottersack, aber vitalem Embryo allzu pessimistische Äußerungen gegenüber der Schwangeren unterbleiben und eher ein Kontrolltermin innerhalb einer Woche vereinbart werden (• Abb. 8).

Eine ausgesprochen echogene Randstruktur des Dottersacks mit echoarmem Inhalt sollte zuerst einmal zu einer Überprüfung des Preset und des Gain am Ultraschallgerät führen. Der prognostische Wert eines sehr echogenen Rands ist umstritten. Dasselbe gilt auch für entrundete, irregulär geformte Dottersäcke (• Abb. 9). Man sieht sie gehäuft bei Abortgeschehen, bei der Verwendung als Prognosefaktoren bei vitalem Embryo wird aber zur Zurückhaltung gemahnt $[1,8]$.

Mit „verkalkter Dottersack“ wird eine Struktur beschrieben, die einem Dottersack entspricht, aber ausgesprochen echodens und „weiß wie Knochen“ ist. Diese wurde bisher nur gleichzeitig mit einem abgestorbenen und ebenfalls sehr echodensen Embryo beschrieben (• Abb. 10)

\section{Kein Doppler am Dottersack!}

Abschließend sei an dieser Stelle noch davor gewarnt, im Zuge von Routineuntersuchungen in der Frühschwangerschaft am Dottersack mit dem Dopplerultraschall herumzuspielen und zu versuchen, farbenprächtige Bilder zu generieren. Der Dottersack spielt eine bisher weitgehend noch nicht ganz verstandene, aber wichtige Rolle bei der frühen Erythropoese. Der Dopplerultraschall setzt diese empfindliche Struktur unnötig hohen Energien aus. Alle wichtigen klinischen Informationen, die man zum Dottersack braucht, kann man mit dem konventionellen B-Mode und 3-D-Schall mit richtiger Einstellung und richtigen Presets bekommen (• Abb. 11). Wer den Dottersack dopplert, verstößt gegen alle einschlägigen Empfehlungen der interna-

Hier steht eine Anzeige. 
tionalen Ultraschall-Safety-Committees [6]!

\section{Fazit für die Praxis}

- Der Dottersack ist die erste embryonale Struktur, die man im Ultraschall in der Frühschwangerschaft erkennen kann.

- Das Vorhandensein eines Dottersacks soll dokumentiert werden, der Durchmesser gemessen und ebenfalls dokumentiert werden.

- Es ist darauf zu achten, dass Dottersack und Scheitel-Steiß-Länge des Embryos getrennt gemessen werden.

- Bei Mehrlingen ist früh auf die Anzahl der Dottersäcke zu achten und diese zu dokumentieren.

- Doppler-Ultraschall des Dottersacks sollte unterbleiben.

- Auffälligkeiten bei Form und Größe des Dottersacks sind mit ungünstigem Schwangerschaftsausgang assoziiert. Sie sollten dokumentiert werden, jedoch keinesfalls dazu führen, dass bei vitalem Embryo gegenüber der Schwangeren sehr pessimistische Prognosen gemacht werden.

\section{Korrespondenzadresse}

\section{Ao. Univ.-Prof. Dr. Christoph Brezinka} Universitätsklinik für gynäkologische Endokrinologie und Reproduktionsmedizin, Medizinische Universität Innsbruck Anichstraße 35, 6020 Innsbruck, Österreich christoph.brezinka@i-med.ac.at

Funding. Open access funding provided by University of Innsbruck and Medical University of Innsbruck.

\section{Einhaltung ethischer Richtlinien}

Interessenkonflikt. C. Brezinka gibt an, dass kein Interessenkonflikt besteht

Für diesen Beitrag wurden vom Autor keine Studien an Menschen oder Tieren durchgeführt. Für die aufgeführten Studien gelten die jeweils dort angegebenen ethischen Richtlinien.

Open Access. Dieser Artikel wird unter der Creative Commons Namensnennung 4.0 International Lizenz (http://creativecommons.org/licenses/by/4.0/deed. de) veröffentlicht, welche die Nutzung, Vervielfältigung, Bearbeitung, Verbreitung und Wiedergabe in jeglichem Medium und Format erlaubt, sofern Sie den/die ursprünglichen Autor(en) und die Quelle ordnungsgemäßnennen, einen Linkzur Creative Commons Lizenz beifügen und angeben, ob Änderungen vorgenommen wurden.

Hinweis des Verlags. Der Verlag bleibt in Hinblick auf geografische Zuordnungen und Gebietsbezeichnungen in veröffentlichten Karten und Institutsadressen neutral.

\section{Literatur}

1. Ashoush S, Abuelghar W, Tamara T, Aljobboury D (2016) Relation between types of yolk sac abnormalities and early embryonic morphology in first-trimester missed miscarriage. J Obstet Gynaecol Res 42:21-28

2. Fenton C, Reidy K, Demyanenko M, Palma-Dias R, Cole S, Umstad MP (2019) The significance of yolk sac number in monoamniotic twins. Fetal Diagn Ther 14:1-7.https://doi.org/10.1159/000496204

3. Golub R, Cumano A (2013) Embryonic hematopoiesis. Blood Cells Mol Dis 51:226-231

4. Odland KH, Johnsen SL, Rasmussen S, Trae G, Reistad HMT, Kiserud T (2019) The human yolk sac size reflects involvement in embryonic and fetal growth regulation. Acta Obstet Gynecol Scand 98:176-182

5. Preisler J, Kopeika J, Ismail L, Vathanan V, Farren J, Abdallah Y, Battacharjee P, Van HC, Bottomley C, Gould D, Johnson S, Stalder C, Van CB, Hamilton J, Timmerman D, Bourne T (2015) Defining safe criteria to diagnose miscarriage: prospective observational multicentre study. BMJ 351:h4579

6. Salvesen K, Lees C, Abramowicz J, Brezinka C, Ter Haar G, Maršál K, Board of International Society of Ultrasound in Obstetrics and Gynecology (ISUOG) (2011) ISUOG statement on the safe use of Doppler in the 11 to $13+6$-week fetal ultrasound examination. Ultrasound ObstetGynecol 37(6):628

7. Suguna B, Sukanya K (2018) Yolk sac size \& shape as predictors of first trimester pregnancy outcome: a prospective observational study. J Gynecol Obstet Hum Reprod 48(3):159-164

8. Tan S, Ipek A, Pektas MK, Arifoglu M, Teber MA, Karaoglanoglu M (2011) Irregular yolk sac shape: is it really associated with an increased risk of spontaneous abortion? J Ultrasound Med 30:31-36

9. Tan S, Pektas MK, Ozcan AS, Akcay Y, Ozat M, Arslan H (2012) Frequency of a persistent yolk sac and its relationship with the gestational outcome. JUltrasound Med 31:697-702

10. Yoneda S, Shiozaki A, Yoneda N, Sameshima A, Ito M, Shima T, Nakashima A, Yoshino O, Kigawa M, Takamori R, Shinagawa Y, Saito S (2018) A yolk sac larger than $5 \mathrm{~mm}$ suggests an abnormal fetal karyotype, whereas an absent embryo indicates a normal fetal karyotype. J Ultrasound Med 37:1233-1241

\section{Kniesehne als Netzersatz bei Gebärmuttersenkung}

Mit einer Sehne aus dem eigenen Knie ist am Universitätsklinikum Mannheim weltweit zum ersten Mal eine Patientin mit Gebärmutter-Senkung behandelt worden. PD Dr. med. Amadeus Hornemann, Oberarzt und Spezialist für minimal-invasive gynäkologische Chirurgie an der Frauenklinik, hat diese neuartige Operation erstmals durchgeführt. Mit dem Verfahren sollen mögliche Komplikationen durch üblicherweise verwendete Kunststoffmaterial vermieden werden.

„Gebärmutter-Senkungen treten im höheren Alter häufig auf", berichtet Hornemann und erklärt: „Sie führen häufig zu Unterleibsschmerzen und Blasenentleerungsstörungen, in schweren Fällen können sogar Teile der Gebärmutter durch die Scheide austreten." Bisher werden solche Senkungen meist durch eine große Bauchoperation, bei der die Gebärmutter mit Kunststoffnetzen an der Wirbelsäule oder den Beckenbändern befestigt wird, behoben. Mit dieser etablierten Methode lassen sich die Beschwerden meist dauerhaft beheben - allerdings treten in seltenen Fällen Unverträglichkeiten des Kunststoff-Netzes auf und das bereits eingewachsene Netz muss in einem komplexen Eingriff wieder entfernt werden.

Um diese Unverträglichkeiten künftig ausschließen zu können, hat Hornemann die neuartige Sehnen-Transplantation entwickelt. Dabei wird eine Sehne aus der Kniekehle entnommen. Anschließend wird sie in einem minimalinvasiven Eingriff an Stelle des bisher üblichen Kunststoffnetzes im Bauchraum zur Fixierung der Gebärmutter verwendet. „Körpereigenes Gewebe wird immer gut vertragen", betont Hornemann. „Daher nutzen wir statt Kunststoff eine Sehne, die schon seit Jahrzehnten bei orthopädischen Eingriffen als Ersatz für defekte Kreuzbänder verwendet wird." Die verwendete Sehne hat keine wesentliche Funktion und spielt für Stabilität und Kraft des Knies keine Rolle.

Quelle: Universitätsmedizin Mannheim, www.umm.de 